\title{
GABAergic and glycinergic inputs to the rabbit oculomotor nucleus with special emphasis on the medial rectus subdivision
}

\author{
P.R. Wentzel, N.M. Gerrits, C.I. de Zeeuw * \\ Department of Anatomy, Erasmus Unilersity Rotterdam, P.O. Box 1738, 3000 DR Rotterdam, The Netherlands
}

Accepted 31 October 1995

\begin{abstract}
Contradictory results have been reported about the inhibitory input to the medial rectus subdivision of the oculomotor nucleus of the cat. In the present ultrastructural study, we quantified the GABAergic and glycinergic terminals in the various subdivisions of the rabbit oculomotor nucleus with the use of post-embedding immunocytochemistry combined with retrograde tracing of horseradish peroxidase. The density of the GABAergic input to the medial rectus subdivision was as substantial as that to the other subdivisions and the postsynaptic distribution of the GABAergic and glycinergic innervation did not differ among the different oculomotor subdivisions.
\end{abstract}

Keywords: Postembedding immunocytochemistry; Electron microscopy; Vestibular nuclei; Eye movement; Vestibulo-ocular reflex

GABA is the main inhibitory transmitter in the vestibular pathways to the oculomotor complex of several species $[4,11,16,18,19]$. The larger part of this GABAergic input is mainly derived from the superior vestibular nucleus (SVN), which seems to subserve vestibular control over all subdivisions of the oculomotor nucleus (OMN) except the medial rectus (MR) motoneurons $[3,16]$. Inhibitory vestibular control over MR motoneurons is maintained through a projection from inhibitory medial vestibular nucleus (MVN) neurons to internuclear cells located in the ipsilateral abducens nucleus (AN) which, in turn, excite the contralateral MR motoneurons [2,3,7]. The inhibitory neurotransmitter involved in this projection to the abducens nucleus is probably glycine $[18,20]$. Thus, while the inhibitory input to oculomotor nucleus and trochlear nucleus is predominantly GABAergic $[4,16,20,22,23]$, the

\footnotetext{
Abbreviations: AN, abducens nucleus; DAB, diaminobenzidine; GABA, $\gamma$-aminobutyric acid; HRP, horseradish peroxidase; IO, inferior oblique; IR, inferior rectus; MR, medial rectus; MVN, medial vestibular nucleus; OMN, oculomotor nucleus; PAP, peroxidase anti peroxidase antibody; PBS, phosphate buffered saline; PhaL, phaseolus vulgaris leucoagglutinin; TBS, Tris-buffered saline; TBST, Triton $(0.1 \%)$ in Trisbuffered saline; TMB, tetramethylbenzidine; SR, superior rectus; SVN, superior vestibular nucleus; WGA-HRP, wheatgerm agglutinated horseradish peroxidase

* Corresponding author. Fax: (31) (10) 436-5780.
}

abducens nucleus (AN) contains more glycinergic than GABAergic boutons [20].

Contradictory results have been reported with respect to the distribution of the GABAergic innervation among the different oculomotor subdivisions of the cat. Spencer et al. [18] reported a lack of GABA in the MR subdivision, whereas De La Cruz et al. [4] showed that the density and the postsynaptic distribution of the GABAergic terminals in the MR region in this species was not significantly different from other OMN subdivisions. In the present study we investigated and compared the GABAergic and glycinergic input to the different subdivisions of the rabbit OMN at the ultrastructural level using postembedding immunocyto-chemistry. Special emphasis was put on the MR subdivision, the motoneurons of which were retrogradely labeled with horseradish peroxidase (HRP) from the MR muscle.

HRP was injected in the MR muscle of four adult pigmented Dutch belted rabbits in order to demarcate the MR motoneurons that are typically distributed between the IR and the IO and SR subdivisions [1,15]. Subsequently, the tissue was processed for electron microscopy and treated for GABA and glycine immunocytochemistry.

General anaesthesia was maintained with a halothaneoxygen mixture (Fluotec Mark II) administered through an endotracheal tube. The MR muscle was injected with 1-5 $\mu 1$ of HRP ( $30 \%$ in saline Miles) with the use of a Hamilton syringe. We reduced the spread of HRP by 
isolating the muscle or by reducing the volume injected (for details, see [15]). After a survival time of 2 days, the animals were premedicated with Hypnorm $(0.25 \mathrm{ml} / \mathrm{kg}$ i.p.), deeply anaesthetized with sodium pentobarbital (Nembutal; $60 \mathrm{mg} / \mathrm{kg}$ i.p.) and perfused with $100 \mathrm{ml}$ saline followed by 21 of $5 \%$ glutaraldehyde in PB. The dissected brains were kept in the same fixative for $1 \mathrm{~h}$. Tissue containing the OMN was cut on a vibratome in 70 $\mu \mathrm{m}$ sections. These sections were collected in PBS, incubated with TMB and $\mathrm{H}_{2} \mathrm{O}_{2}$, stabilized with $\mathrm{DAB}$ in a cobalt solution $[5,12]$, rinsed in a glucose solution in $\mathbf{P B}$, postfixed for $1 \mathrm{~h}$ in $1 \%$ osmium tetroxide in the same solution and rinsed in PB and distilled water. Subsequently, these sections were chemically dehydrated with acidified dimethoxy-propane [14] and flat embedded in araldite on slides between polyethyleen foils. Ultrathin sections were cut from pyramids of the OMN on a Reichert ultratoom and mounted on formvar coated nickel grids. The grids were rinsed in a solution of Tris buffer containing $0.9 \% \mathrm{NaCl}$ and $0.1 \%$ Triton-X100 at $\mathrm{pH} 7.6$ (TBST) and incubated overnight in a droplet of GABA (1:2,000 in TBST) or glycine (1:250 in TBST) antiserum. The GABA and glycine antisera were generously supplied by Dr. R.M. Buijs and Dr. R.J. Wenthold, respectively (for specificity tests, see $[17,21])$. The next day the grids were rinsed twice with TBST and stored for 30 minutes in the rinsing solution. After rinsing with TBST ( $\mathrm{pH}$ 8.2) the grids were incubated for $1 \mathrm{~h}$ in a droplet of goat anti rabbit IgG labeled with $15 \mathrm{~nm}$ gold particles (Janssen Pharm.) diluted 1:25 in TBST. Subsequently, the grids were rinsed with TBST (pH 7.6) and distilled water, counterstained with uranyl acetate and lead citrate and examined in a Philips electron microscope.

Pyramids of the OMN ipsilateral to the injected eye muscle were made. The tissue was cut in such a way that they contained besides the MR subdivisions also either the IR or IO subdivision, or both the IO and SR subdivisions. For each OMN, ultrathin sections were made from at least two pyramids of different rostrocaudal levels. At least two non-serial ultrathin sections from each pyramid were analyzed for GABA-immunoreactivity, while another nonserial section of these pyramids was analyzed for glycineimmunoreactivity. The boundaries of the respective subdivisions were determined from photographs of semithin (2 $\mu \mathrm{m})$ sections. To avoid mistakes in the identification of the borders of the subdivisions we used a safety margin of approximately $100 \mu \mathrm{m}$ from each border in which no terminals were counted for analysis. Within the boundaries we randomly sampled the presence of GABA and glycine immunopositive terminals as well as the non-labeled terminals. A terminal was considered GABA positive or glycine positive, when the number of gold particles overlying it was at least eight times higher than the number of particles overlying an equal surface area of surrounding non-labeled structures (for details about counting procedure, see $[6,9]$ ).

Injection of HRP in the MR muscle resulted in strong labeling of motoneuronal cellbodies throughout the rostrocaudal extent of the ipsilateral OMN. The HRP labeled cells in the caudal part of the OMN were arranged in an
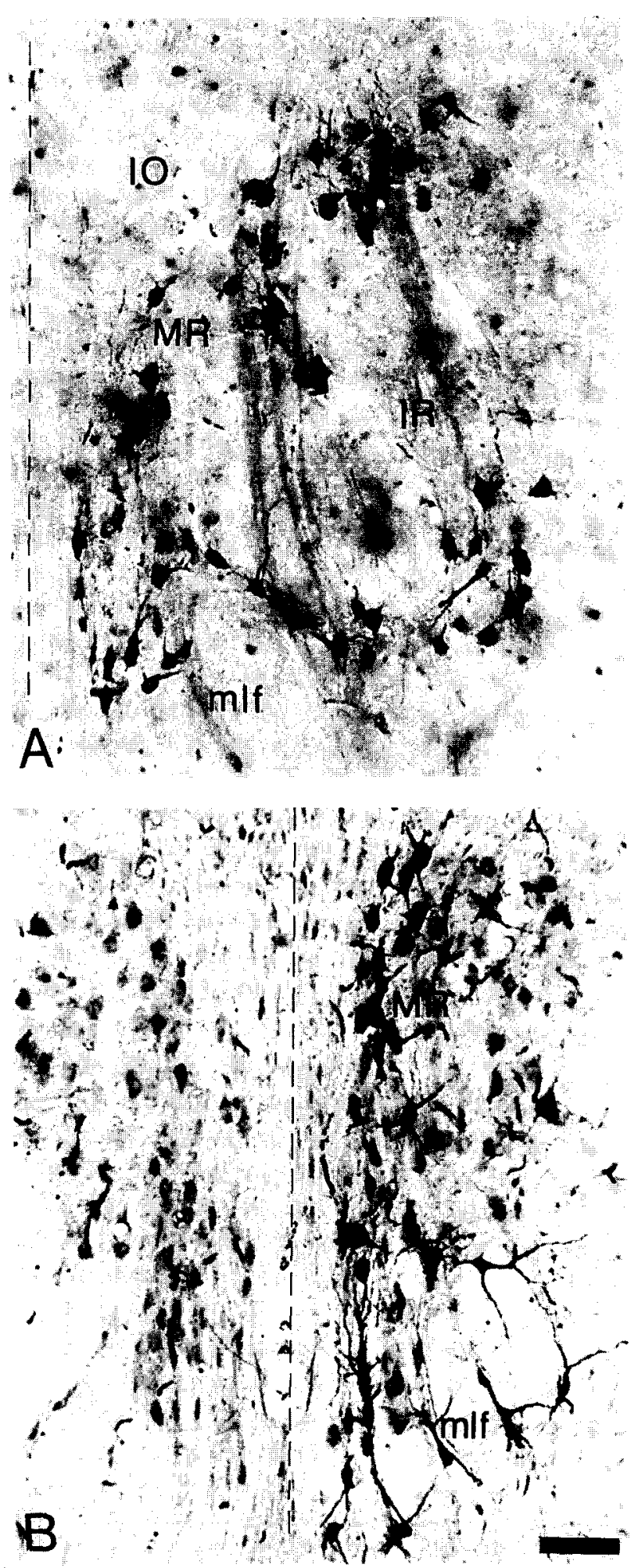

Fig. 1. A: caudal part of the OMN showing the dorsolateral to ventromedial and the ventrolateral to ventromedial bands of $\mathrm{MR}$ motoneurons, labeled with HRP from the ipsilateral MR muscle. B: photomicrograph of the more rostral part of the OMN showing the dorsolateral to ventromedial band of HRP-labeled MR motoneurons. Bar equals $0.1 \mathrm{~mm}$. 
oblique band that extended from the ventromedial to the dorsolateral borders of the OMN (Fig. 1A). In addition, many labeled cells were found among the fibres of the medial longitudinal fasciculus (MLF). More rostrally, the labeled motoneurons approximate the midline of the OMN (Fig. 1B). The semithin sections used to delineate the boundaries between the oculomotor subdivisions showed the same pattern.

GABA immunopositive terminals ranged from 1 to 4 $\mu \mathrm{m}$ in diameter and contained many mitochondria. Their vesicles were predominantly flattened and their synaptic contacts were usually multiple and symmetric (F-type [10], Fig. 2). In all oculomotor subdivisions approximately $50 \%$ of the encountered axon terminals were GABA positive (Table 1). No major differences were observed between the subdivisions. In all subdivisions, the GABAergic terminals were similarly distributed within the neuropil: on average $9.5 \%$ of the terminals contacted somata and $20.2 \%$ and $70.3 \%$ contacted proximal and distal dendrites, respectively (Tables 2 and 3; Fig. 2). Sometimes, the GABAergic and non-GABAergic terminals were apposed to spines that arose from cellbodies or dendrites (Fig. 3). These spines
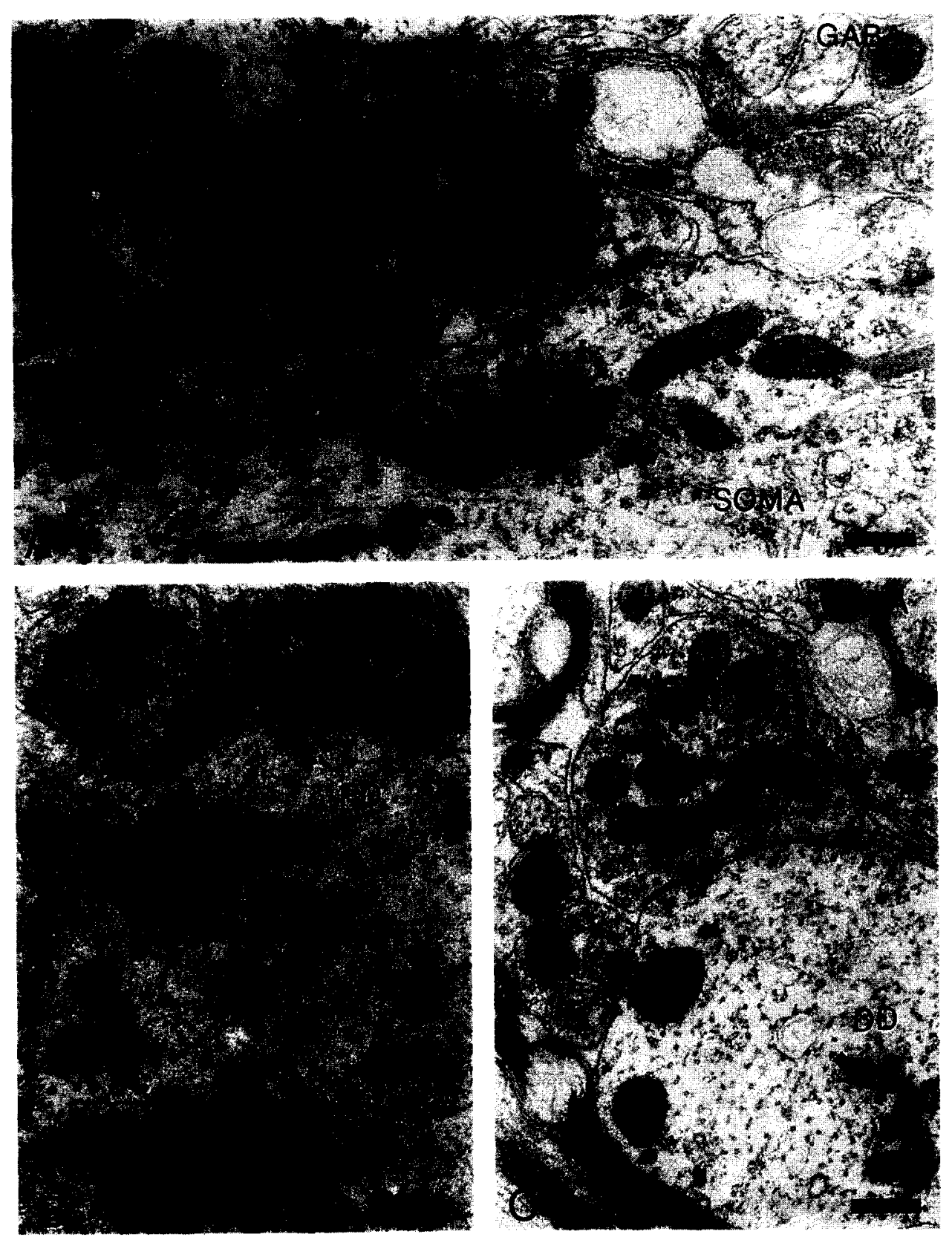

Fig. 2. Electron micrographs of GABA-positive axon terminals adjacent to HRP-labeled structures in the MR region of the OMN. Gold particles (diameter equals $15 \mathrm{~nm}$ ) indicate the presence of GABA. Synaptic contacts and HRP/DAB-Cobalt reaction product are indicated by arrowheads and arrows, respectively. The axon terminals contain small flattened vesicles and establish symmetric synaptic contacts. Panel A illustrates a contact with a soma; B demonstrates a contact with a large proximal dendrite (PD) and $\mathrm{C}$ a synaptic contact with a small dendrite (DD). Bar equals $0.5 \mu \mathrm{m}$. 
Table 1

Percentages of immunopositive and immunonegative, GABAergic (A) and glycinergic (B) terminals $(n=4878)$ in different oculomotor subdivisions

\begin{tabular}{llllll}
\hline A & $\begin{array}{l}\text { GABA }+ \\
(\%)\end{array}$ & $\begin{array}{l}\text { GABA- } \\
(\%)\end{array}$ & B & $\begin{array}{l}\text { gly }+ \\
(\%)\end{array}$ & $\begin{array}{l}\text { gly - } \\
(\%)\end{array}$ \\
\hline MR & 48 & 52 & MR & 4 & 96 \\
IR & 52 & 48 & IR & 5 & 95 \\
IO & 45 & 55 & IO & 3 & 97 \\
SR & 49 & 51 & SR & 4 & 96 \\
\hline
\end{tabular}

sometimes showed accumulations of clear and coated vesicles in the spine head and the interneuronal cleft was unusually straight.

In all subdivisions only few terminals were glycinergic (3-5\%; Table 1). The labeling of the glycinergic profiles differed somewhat from that of the GABAergic profiles. The glycinergic axons usually contained a larger number of gold particles than the GABAergic axons, while the glycinergic terminals usually carried fewer gold particles. The morphological characteristics of the glycinergic and GABAergic profiles were similar. The glycine immunoreactive terminals in the $\mathrm{OMN}$ were small to medium sized F-types with many mitochondria and symmetric synaptic contacts (Fig. 4). In all subdivisions the distribution of the glycinergic terminals in the neuropil was similar to that of the GABAergic terminals (Tables 2 and 3). Most of the glycinergic terminals were apposed to distal dendrites $(74.5 \%$ ), while $9.6 \%$ and $16.9 \%$ were apposed to somata and proximal dendrites, respectively.

To find out whether different neurons within the MR subdivision may receive different inputs we compared the synaptic input of motoneurons retrogradely labeled with HRP from the MR muscle with that of non-labeled neurons in the MR subdivision. The percentage of GABA positive $(43.3 \%)$ and glycine positive $(7.7 \%)$ boutons contacting motoneurons $(n=50)$ that were retrogradely labeled with HRP was similar to that of GABA positive (39.7\%) and glycine positive $(6.4 \%)$ boutons contacting non-labeled somata $(n=50)$. These data suggest that the synaptic input of motoneurons is not substantially different from that of the non-motoneurons of the oculomotor complex.

In the present study we demonstrated that MR motoneurons of the rabbit oculomotor nucleus receive a prominent GABAergic input. The density and postsynaptic distribution of the GABAergic innervation in the medial rectus motoneuron group was similar to that of the other subdivisions of the OMN and the morphological characteristics of the GABAergic synaptic endings in the different subdivisions were the same. These observations are in agreement

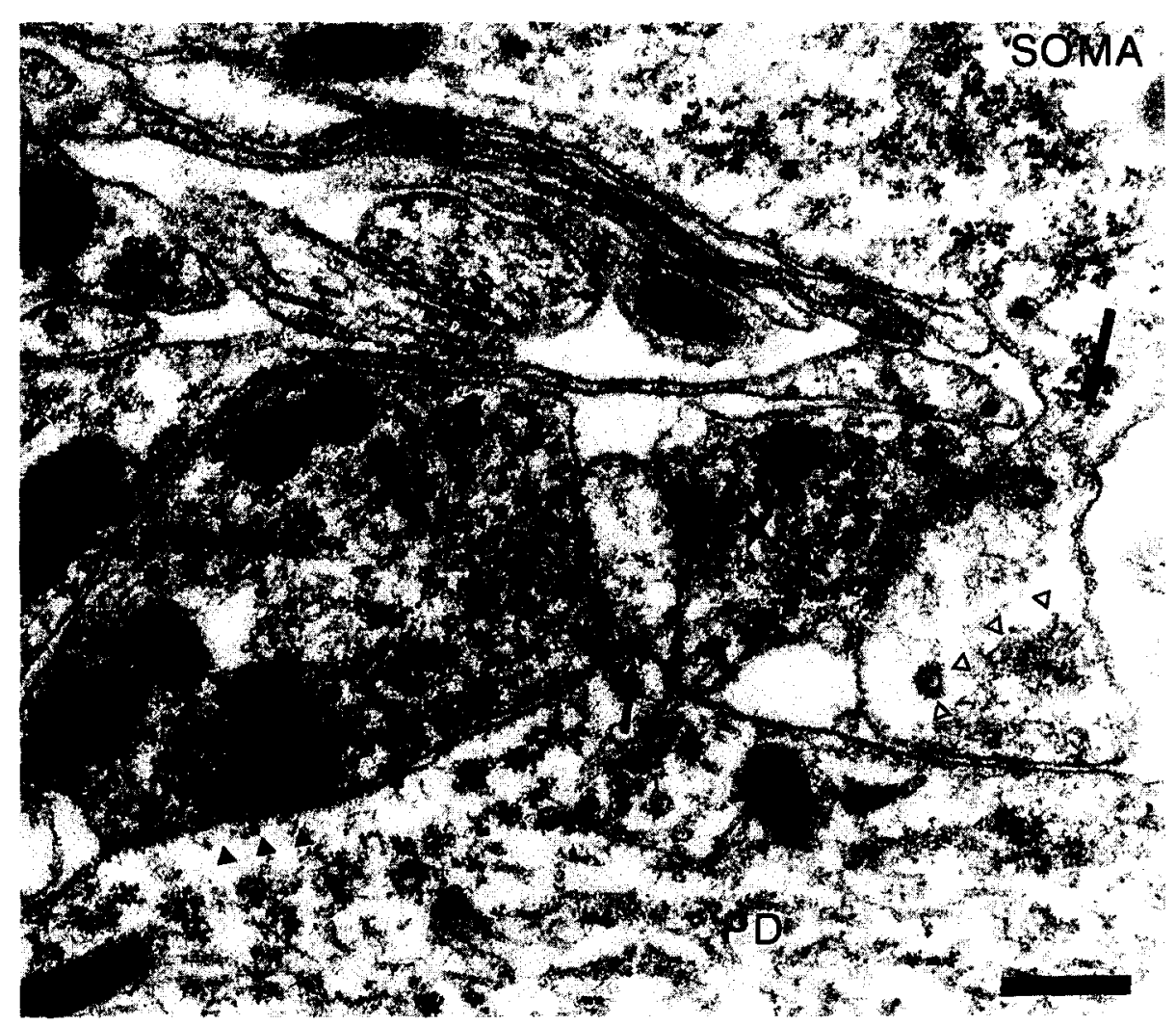

Fig. 3. Electron micrograph of a GABA positive terminal (asterisk) and an unlabeled terminal (star) that make synaptic contacts (arrowheads) with an unlabeled proximal dendrite (PD) and dendritic spine (small arrow), respectively. The large arrow indicates the entrance of a somatic spine that is apposed to the same PD. The open arrowheads point at the vesicles accumulated in the spinehead. Bar equals $0.5 \mu \mathrm{m}$. 
Table 2

Postsynaptic distribution of randomly sampled GABA and glycine immunoreactive terminals

\begin{tabular}{llllll}
\hline & & & $\begin{array}{l}\text { Soma } \\
(\%)\end{array}$ & $\begin{array}{l}\text { Prox. dendr. } \\
(\%)\end{array}$ & $\begin{array}{l}\text { Dist. dendr. } \\
(\%)\end{array}$ \\
\hline MR & GABA + & $n=384$ & 9.8 & 18.8 & 71.4 \\
& GABA - & $n=416$ & 9.6 & 26.0 & 64.4 \\
& glycine + & $n=15$ & 6.7 & 13.3 & 80.0 \\
IR & glycine - & $n=364$ & 9.9 & 20.0 & 70.1 \\
& GABA + & $n=416$ & 8.7 & 20.7 & 70.6 \\
& GABA - & $n=384$ & 7.3 & 15.6 & 77.1 \\
& glycine + & $n=23$ & 4.3 & 17.4 & 78.3 \\
IO & glucine - & $n=443$ & 9.0 & 22.1 & 68.9 \\
& GABA + & $n=360$ & 9.4 & 17.3 & 73.3 \\
& GABA - & $n=440$ & 9.3 & 24.1 & 66.6 \\
& glycine + & $n=13$ & 15.4 & 15.4 & 69.2 \\
& glycine - & $n=361$ & 8.3 & 22.7 & 69.0 \\
SR & GABA + & $n=392$ & 10.2 & 24.0 & 65.8 \\
& GABA - & $n=408$ & 11.7 & 21.6 & 66.7 \\
& glycine + & $n=17$ & 11.8 & 17.6 & 70.6 \\
& glycine - & $n=396$ & 9.6 & 22.0 & 68.4 \\
\hline
\end{tabular}

It was determined for each oculomotor subdivision what percentage of the terminals contacted somata, and proximal or distal dendrites.

with the findings of De La Cruz et al. [4] in the oculomotor nucleus of the cat.

The major source of the GABAergic innervation of the $M R$ region remains to be determined. The $S V N$ gives rise to a small part of the GABAergic input to the MR subdivision [16], but it has not been established whether these terminals innervate MR motoneurons, MR interneurons or dendrites of motoneurons of adjacent subdivisions that extend into the MR subdivision. The direct input from the SVN to MR motoneurons is probably not very substantial, since IPSP's have not been found in MR motoneurons following vestibular stimulation $[3,8]$. Possible sources for the GABAergic input to the MR motoneurons include the pontine and mesencephalic reticular formation, prepositus hypoglossi nucleus and oculomotor internuclear neurons (for a review, see $[4,13]$ ).

An extensive immunocytochemical, electrophysiological-pharmacological and autoradiographic study in the cat [20] has revealed that glycine is utilized in inhibitory connections from the prepositus hypoglossi nucleus, parts

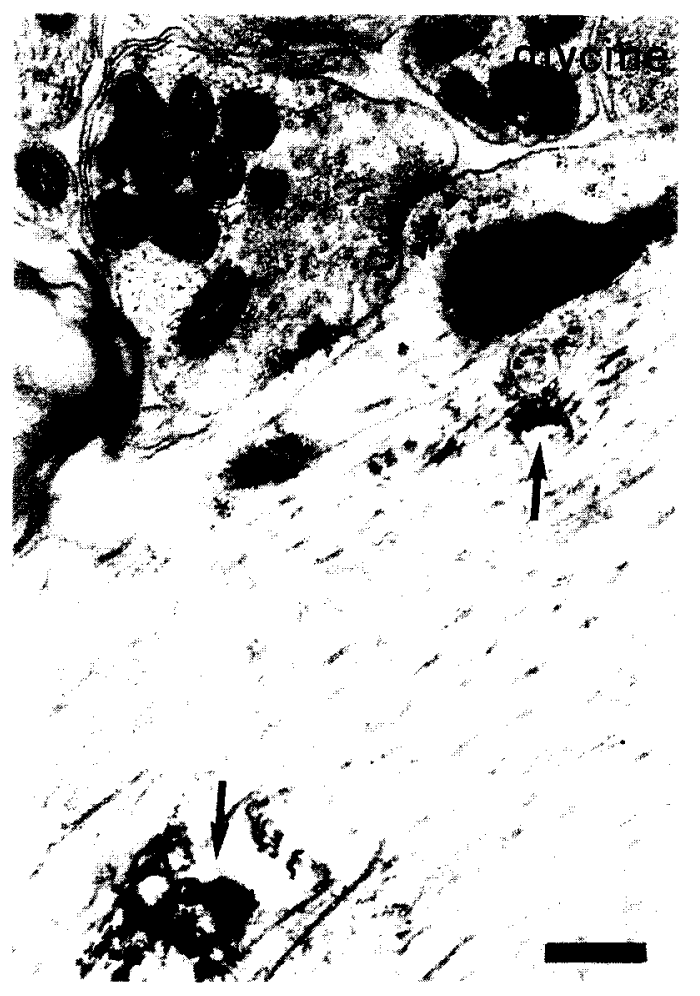

Fig. 4. Electron micrograph of a glycine positive axon terminal contacting a HRP labeled proximal dendrite. Gold particles indicate the presence of glycine. Arrowheads indicate synaptic contacts; arrows indicate $\mathrm{HRP} / \mathrm{DAB}$-Cobalt reaction product. Bar equals $0.5 \mu \mathrm{m}$.

of the reticular formation and second-order vestibular neurons to the abducens nucleus. Thus, while GABA may control mainly the inhibition of vertical eye movements, glycine is probably the major inhibitory transmitter of horizontal eye movements. In agreement with studies in the cat and monkey $[18,20]$, the OMN of the rabbit contains only few glycine-immunoreactive boutons. In contrast to these studies, however, the glycine-immunoreactive boutons in the OMN of the rabbit are distributed through all subdivisions including the MR region. The source and functional implications of this innervation remains unclear. Considering the low occurrence of glycine in the OMN and the fact that most, if not all, of the glycine immuno-

Table 3

Presynaptic distribution of randomly sampled somata and proximal dendrites in the different oculomotor subdivisions

\begin{tabular}{llllllll}
\hline A & & GABA $+(\%)$ & GABA $-(\%)$ & B & & gly $+(\%)$ & gly $-(\%)$ \\
\hline MR & soma & 43.3 & 56.7 & MR & soma & 7.7 & 92.3 \\
& prox. den & 48.5 & 51.5 & & prox. den & 13.2 & 86.8 \\
IR & soma & 38.8 & 61.2 & IR & soma & 5.4 & 94.6 \\
& prox. den & 62.4 & 37.6 & & prox. den & 11.2 & 88.8 \\
IO & soma & 41.2 & 58.8 & IO & soma & 4.7 & 95.3 \\
& prox. den & 70.6 & 29.4 & SR & prox. den & 14.7 & 85.3 \\
SR & soma & 44.5 & 55.5 & & soma & 10.2 & 89.8 \\
& prox. den & 66.6 & 33.4 & prox. den & 10.3 & 89.7 \\
\hline
\end{tabular}

In the sections processed for GABA (A) and glycine (B) immunocytochemistry of each subdivision we analyzed the terminals on 50 somata and 100 proximal dendrites. 
reactive terminals also contain GABA [22], we cannot exclude the possibility that the function of glycine is merely metabolic.

\section{Acknowledgements}

We are grateful to Dr. T.J.H. Ruigrok for his expert help during surgical procedures. The excellent technical assistance of Mr. J. van der Burg, Mrs. H. Sabel-Goedknegt, Mr. R. Hawkins and Mr. E. Dalm is also greatly appreciated.

\section{References}

[1] Akagi, Y., The localization of the motor neurons innervating the extraocular muscles in the oculomotor nuclei of the cat and rabbit, using horseradish peroxidase, J. Comp Neurol., 181 (1978) 745-762.

[2] Baker, R., Grestly, M. and Berthoz, A., Neuronal activity in the prepositus hypoglossi nucleus correlated with vertical eye movement in the cat, Brain Res., 101 (1975) 366-371.

[3] Baker, R. and Highstein, S.M., Vestibular projections to medial rectus subdivision of oculomotor nucleus, J. Neurophysiol., 41 (1978) 1629-1646.

[4] De La Cruz, R.R., Pastor, A.M., Martínez-Guijarro, F.J., LópezGarcía, C. and Delgado-García, J.M., Role of GABA in the extraocular motor nuclei of the cat: a postembedding immunocytochemical study, Neuroscience, 51 (1992) 911-929.

[5] De Zeeuw, C.I., Holstege, J.C., Calkoen, F., Ruigrok, T.J.H. and Voogd, J., A new combination of WGA-HRP anterograde tracing and GABA immunocytochemistry applied to afferents of the cat inferior olive at the ultrastructural level, Brain Res., 447 (1988) 369-375.

[6] De Zeeuw, C.I., Holstege, J.C., Ruigrok, T.J.H. and Voogd, J., An ultrastructural study of the GABAergic, the cerebellar and the mesodiencephalic innervation of the cat medial accessory olive: anterograde tracing combined with immunocytochemistry, J. Comp Neurol., 284 (1989) 12-35.

[7] Highstein, S.M. and Baker, R., Excitatory termination of abducens internuclear neurons on medial rectus motoneurons. Relationship to syndrome of internuclear opthalmoplegia, J. Neurophysiol., 41 (1978) 1647-1661.

[8] Highstein, S.M. and Reisine, H., Synaptic and functional organization of vestibulo-ocular pathways. In R.Granit and O. Pompeiano (Ed.), Progress in Brain Research, Elsevier Science, Amsterdam, 1979, pp. 431-442.
[9] Holstege, J.C. and Bongers, C.M., A glycinergic projection from the ventromedial lower brainstem to spinal motoneurons: an ultrastructural double labeling study in the rat, Brain Res., 566 (1991) 308-315.

[10] Holstege, J.C. and Calkoen, F., The distribution of GABA in lumbar motoneuronal cell groups. A quantitative ultrastructural study in rat, Brain Res., 530 (1990) 130-137.

[11] Lanoir, J., Soghomonian, J.J. and Cadenel, G., Radioautographic study of ${ }^{3} \mathrm{H}$-GABA uptake in the oculomotor nucleus of the cat, Exp. Brain Res., 48 (1982) 137-143.

[12] Lemann, W., Saper, C.B., Rye, D.B. and Wainer, B.H., Stabilization of TMB reaction product for electronmicroscopic retrograde and anterograde fiber tracing, Brain Res. Bull., 14 (1985) 277-281.

[13] May, P.J., Baker, H., Vidal, P., Spencer, R.F. and Baker, R., Morphology and distribution of serotonergic and oculomotor internuclear neurons in the cat midbrain, J. Comp Neurol., 266 (1987) $150-170$.

[14] Muller, L.L. and Jacks, T.J., Rapid histochemical dehydration of samples for electron microscopic examinations, J. Histochem. Cytochem., 23 (1975) 107-110.

[15] Murphy, E.H., Garone, M., Tashayyod, D. and Baker, R.B., Innervation of extraocular muscles in the rabbit, J. Comp Neurol., 254 (1986) 78-90.

[16] Sato, Y. and Kawasaki, T., Target neurons of floccular caudal zone inhibition in Y-group nucleus of vestibular nuclear complex, $J$. Neurophysiol., 57 (1987) 460-480.

[17] Seguela, P., Geffard, P., Buijs, R. and Le Moal, M., Anti-bodies against $\tau$-aminobutyric acid: specificity studies and immunocytochemical results, Proc. Natl. Acad. Sci. USA, 81 (1984) 3888-3892.

[18] Spencer, R.F. and Baker, R., GABA and glycine as inhibitory neurotransmitters in the vestibuloocular reflex. In B. Cohen, D.L. Tomko and F. Guedry (Ed.), Sensing and Controlling Motion: Vestibular and Sensorimotor Function, The New York Academy of Sciences, New York, 1992, pp. 602-611.

[19] Spencer, R.F., Wang, S.F. and Baker, R., The pathways and functions of GABA in the oculomotor system. In R.R. Mize, R.E. Marc and A.M. Sillito (Ed.), Progress in Brain Research, Elsevier Science, Amsterdam, 1992, pp. 307-331.

[20] Spencer, R.F., Wenthold, R.J. and Baker, R., Evidence for glycine as an inhibitory neurotransmitter of vestibular, reticular and prepositus hypoglossi neurons that project to the cat abducens nucleus, $J$. Neurosci., 9 (1989) 2718-2736.

[21] Wenthold, R.J., Huie, D., Altschuler, R.A. and Reeks, K.A., Glycine immunoreactivity localized in the cochlear nucleus and the superior olive complex, Neuroscience, 22 (1987) 897-912.

[22] Wentzel, P.R., De Zeeuw, C.I., Holstege, J.C. and Gerrits, N.M., Colocalization of GABA and glycine in the rabbit oculomotor nucleus, Neurosci. Lett., 164 (1993) 25-29.

[23] Wentzel, P.R., De Zeeuw, C.I., Holstege, J.C. and Voogd, J., GABAergic and glycinergic synaptic inputs to the rabbit oculomotor nucleus, Eur. Neurosci. Meeting (Madrid), Suppl. 2, 1054 (1993) 271 (abstracts). 